\title{
A New-Fangled and Alternative Synthesis of Anticoagulant Drug Substance, Apixaban
}

\author{
T. Suresh* and K. Rambabu \\ Department of Chemistry, RVR \& JC College of Engineering, Guntur, A.P, India
}

\section{ABSTRACT}

A cost effective and technically viable process was for apixaban(anticoagulant drug) developed by using commercially available chlorinating agent sulfurylchloride in the place of phosphorus pentachloride not as everyone using and introduced a new amidation process for ester simply by treating with sodamide and sodium methoxide in methylene dichloride to land up with cost effective and simple process.

KEY WORDS: COMMERCIALLY VIABLE PROCESS, APIXABAN, DI-CHLORINATION, SULFURYL CHLORIDE. AMIDATION, SODAMIDE.

\section{INTRODUCTION}

Apixaban (Apixaban. Monograph for Professionals.2019; Raghavan, N., et al., 2009) is an oral anticoagulant used to lower the risk of stroke caused by a blood clot in people with a heart rhythm disorder called atrial fibrillation .It is also used after hip or knee replacement surgery to prevent a type of blood clot called deep vein thrombosis (DVT), which can lead to blood clots in the lungs. There are several routes were available in the literature (Zhou , JC., et al., 2003; Jianan Jiang. and, Yafei, Ji. et al., 2012; Ji, Y., et al., 2012; Huo, SW., et al., 2014;Guo, F., 2014;Dwivedi SD., et al., 2014;Shekar Bhaskar Bhirud., et al., 2014)for its synthesis, but few of were cost effective methods. After a through reveals of the literature of apixaban, the author developed a cost effective and scalable process.

\section{Experimental}

\section{MATERIAL AND METHODS}

All the raw materials used for the selected route of synthesis are procured from Aldrich and the reagent grade

Biosc Biotech Res Comm P-ISSN: 0974-6455 E-ISSN: 2321-4007

\section{crossey}

Identifiers and Pagination

Year: 2021 Vol: 14 No (5) Special Issue

Pages: 248-252

This is an open access article under Creative

Commons License Attribn 4.0 Intl (CC-BY).

DOI: http://dx.doi.org/10.21786/bbrc/14.5/45 materials were purchased from Merck synthesis. All the solvents used for its entire apixaban process development were purchased from Merck inc.

\section{RESULTS AND DISCUSSION}

Most of the literatures reported for chlorination of 1-(4-iodophenyl) piperidin-2-one is in phosphorous pentachloride ( $\mathrm{PCl}$ ) only, but the author in this research invented a new and cheap reagent sulfuryl chloride $(\mathrm{SO} 2 \mathrm{Cl} 2)$ in the place of $\mathrm{PCl}$. Which given fruitful results same like regular di-halogenation. In the selected route of synthesis of apixaban was involved a new sequence is that amidation followed by $\mathrm{N}$-alkylation with piperdin2-one whereas in regular synthesis it is in reverse mode and it has more advantages is that it can be eliminated or minimized the formation of pipridin-2-one ring opening impurity and as well as apixaban acid impurity (Figure 1).

Step-1: Synthesis of 3, 3-Dichloro-1-(4-lodophenyl) Piperidin-2-one: In a 500mL round bottom flask $200 \mathrm{~mL}$ of sulfuryl chloride was taken at room temperature and cooled the reaction mass temperature to 0-5oC. Added $50 \mathrm{gm}$ of 1-(4-iodophenyl) piperidin-2-one portion wise (5 lots) under gentle stirring which lead to the formation of gel type reaction mass and is maintained for 3-4 hours at 0-5 oC. After completion of the TLC (1:9, Ethyl acetate and Hexane) reaction mass was quenched in ice cooled water $(300 \mathrm{~mL})$ and the resulting solution was $\mathrm{pH}$ adjusted to 7.5-8.0 with sodium carbonate and extracted with
Article Information

Received: $19^{\text {th }}$ Jan 2021

ccepted after revision: $27^{\text {th }}$ Mar 2021 
Ethyl acetate $(3 \times 100 \mathrm{~mL})$, the combined organics were washed with brine solution and dried over anhydrous sodium sulfate. The organic layer further distilled off under reduced pressure and obtained solid was filtered with hexanes slurry to get 3, 3-dichloro-1-(4-iodophenyl) piperidin-2-one as white colored solid material $60 \mathrm{gm}$. 1H NMR (CDCl3, ppm): $\delta 2.07-2.13$ (m, 2H), $22.90-2.93$ $(\mathrm{m}, 2 \mathrm{H}) ; 3.72(\mathrm{t}, 2 \mathrm{H}) ; \delta 7.13(\mathrm{~d}, 2 \mathrm{H}) ; \delta 7.77(\mathrm{~d}, 2 \mathrm{H}) ;$ Mass: $370.7(\mathrm{M}+\mathrm{H}), 372(\mathrm{M}+2)$; FT-IR (Cm-1): 3087.93, 2967.07, 2945.89, 1681.72, 1485.27, 1474.87, 685.62, 792.46.

Figure 1: Scheme-I-New route of Synthesis

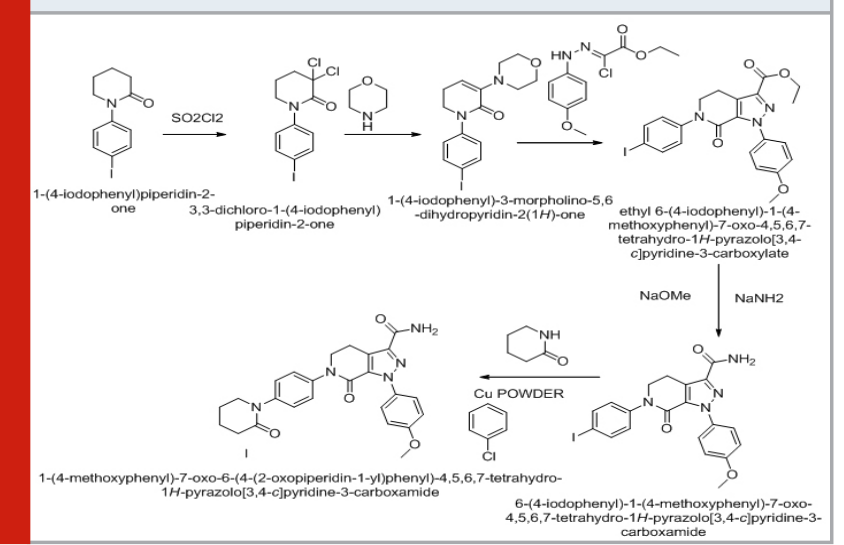

Figure 2: APN-(a) 1HNMR

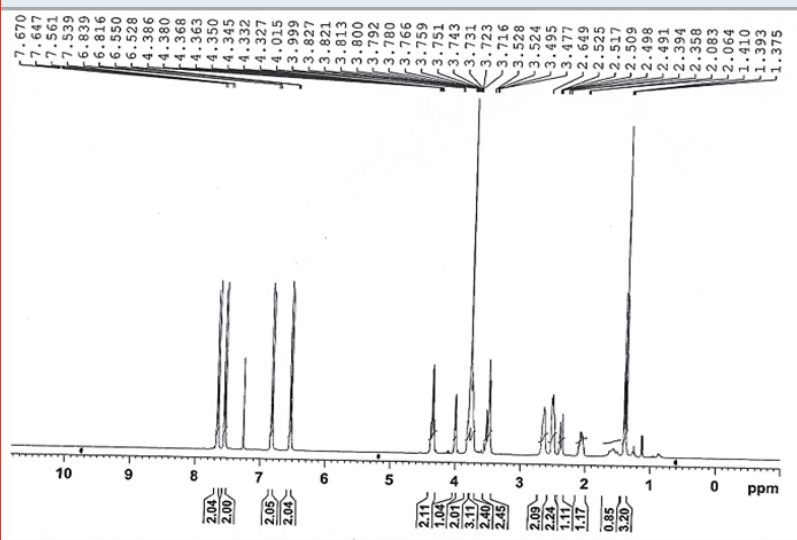

Figure 3: APN-(a): FT-IR

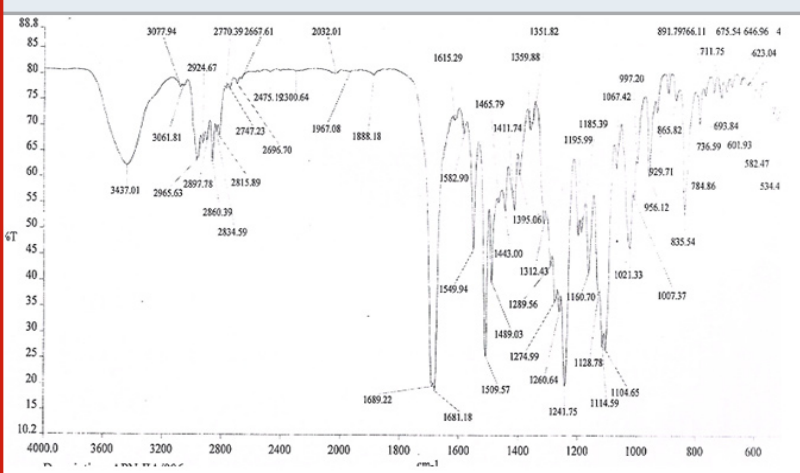

Step-2: Synthesis of 1-(4-lodophenyl)-3-Morpholino-5, 6-Dihydropyridine-2(1h)-One(a): In a dry 250mL round bottom flask 25gm of 3, 3-dichloro-1-(4-iodophenyl) piperidin-2-one was taken in $100 \mathrm{~mL}$ of Ethyl acetate and added 10gm of Tri-ethyl amine followed by addition of $15 \mathrm{gm}$ morpholin in $25 \mathrm{~mL}$ of Ethyl acetate at room temperature and maintain for another 2 hours. After completion of the reaction mass quenched with Water and separated the organic layer, washed with brine solution and dried over sodium sulfate. The organic layer was distilled under reduced pressure and obtained 1-(4-iodophenyl)-3-morpholino-5, 6-dihydropyridine$2(1 \mathrm{H})$-one is dried and packed 30gm.

Figure 4: APN-(a): 13CNMR

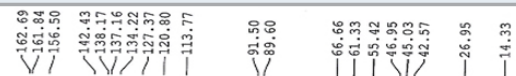

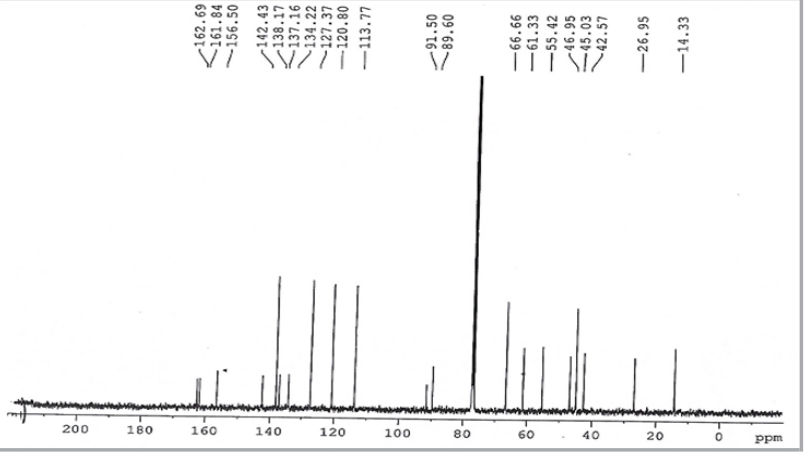

Figure 5: APN-(a): MASS

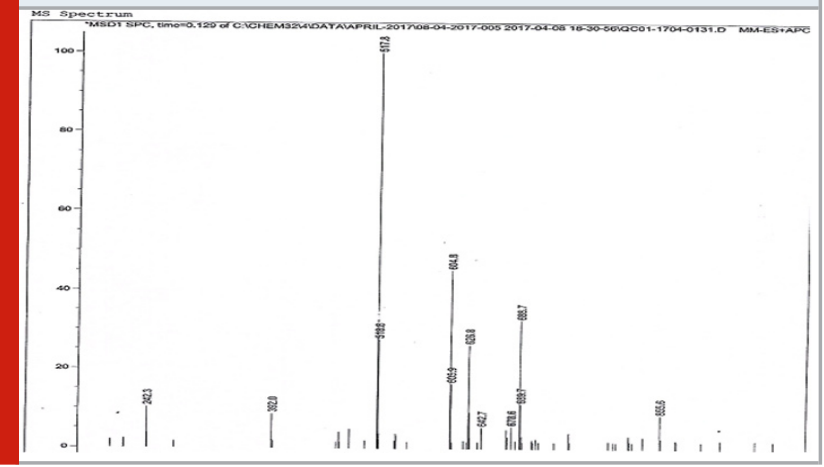

Figure 6: APN-(b) 1HNMR

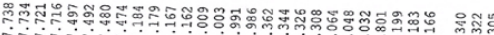
ituviviviving

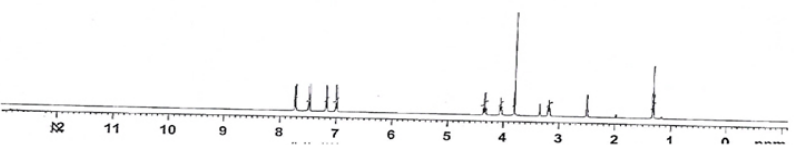

Step-3: Synthesis of Ethyl-6-(4-lodophenyl)-1-(4Methoxyphenyl)-7-0xo-4,5,6,7-Tetrahydro-1 hPyrazolo[3,4c]Pyridine-3-Carboxylate(b): In a dry 500 $\mathrm{mL}$ round bottom flask $50 \mathrm{gm}$ of 1-(4-iodophenyl)-3morpholino-5, 6-dihydropyridine- $2(1 \mathrm{H})$-one and $100 \mathrm{~mL}$ of methylene dichloride was taken at 0-5o $\mathrm{C}$ and added $48 \mathrm{gm}$ of (Z)-Ethyl-2-chloro-2-(2-(4-methoxyphenyl) 
hydrazono)acetate dissolved in $50 \mathrm{~mL}$ of methylene dichloride drop wise and maintain it for 3-4 hours at $0-50 \mathrm{C}$ and then raise the temperature to room temperature, maintain at for another 3 hours. After completion of the reaction $100 \mathrm{~mL}$ of water added and separated the organic layer and removed the solvent under reduced pressure. The resulting crude material was taken in $200 \mathrm{~mL} 4 \mathrm{~N}$ hydrochloride solution and maintained it for 6 hours at room temperature and after completion of TLC; added additional $200 \mathrm{~mL}$ water and stirred for 30minutes at 0-5o C, filtered the resulting precipitated solid, filtered bed was washed with $35 \mathrm{~mL}$ water and dried to afford 42 gm of Ethyl-6-(4-iodophenyl)-1-(4-methoxyphenyl)7-oxo-4, 5, 6, 7-tetrahydro-1H-pyrazolo[3,4c]pyridine3-carboxylate as off-white solid.

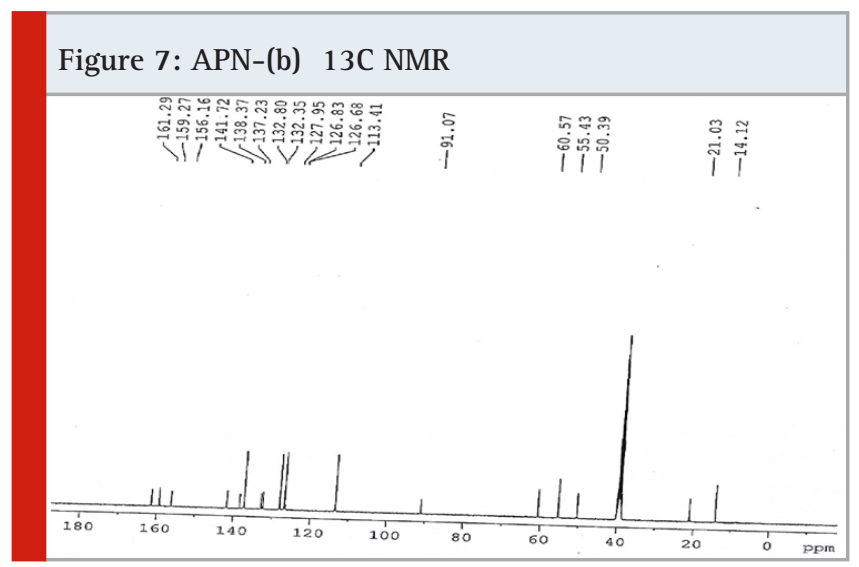

Figure 7: APN-(b) FTIR

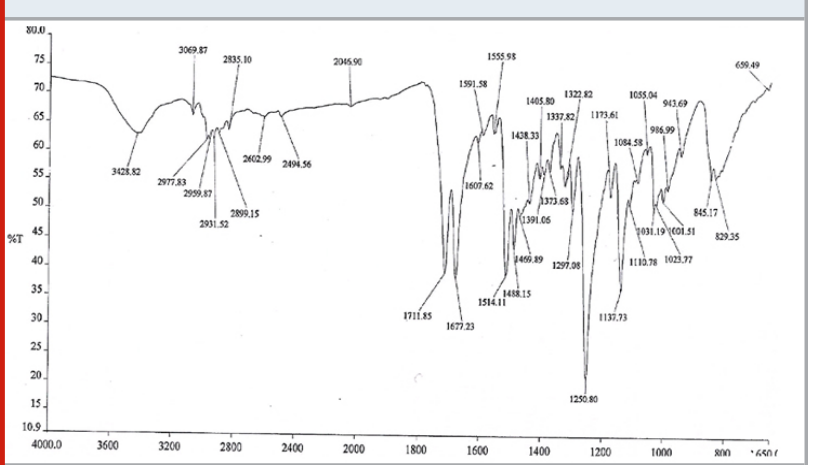

Step-4: Ethyl-1-(4-Methoxyphenyl)-7-0xo-6-(4(2-0xopiperidin-1 yl)Phenyl)-4, 5, 6, 7-Tetrahydro1h-Pyrazolo[3,4c]Pyridine-3-Carboxylate(c): 20gm of Ethyl-6-(4-iodo-phenyl)-1-(4-methoxyphenyl)-7oxo-4, 5, 6, 7-tetrahydro-1H-pyrazolo[3,4c]pyridine3-carboxylate, $25 \mathrm{gm}$ of piperidin-2-one and $5 \mathrm{gm}$ of copper(I)iodide taken in a mixture solution of $40 \mathrm{gm}$ of anhydrous potassium carbonate and $5.0 \mathrm{~mL}$ of triethylamine in $200 \mathrm{~mL}$ of Toluene. The reaction mixture heated for reflux and collected water by maintaining the reaction mixture for 8-9 hours. Once reaction completed over TLC, then filter the reaction mass through celite bed and the resulting filtrate mother liquor was concentrated under reduced pressure to get crude Ethyl1-(4-methoxyphenyl)-7-oxo-6-(4-(2-oxopiperidin-1yl) phenyl)-4, 5, 6, 7-tetrahydro-1H-pyrazolo[3,4c]pyridine- 3-carboxylate and which was isolated in ethyl acetate and hexane (1:1 ratio) to yield pure compound $15 \mathrm{gm}$ with purity $\leq 99 \%$.

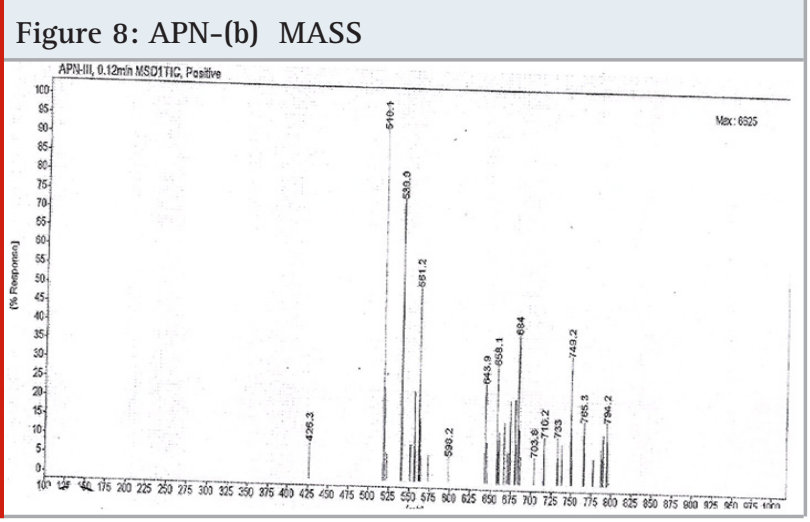

Figure 9: APN-(c) 1HNMR
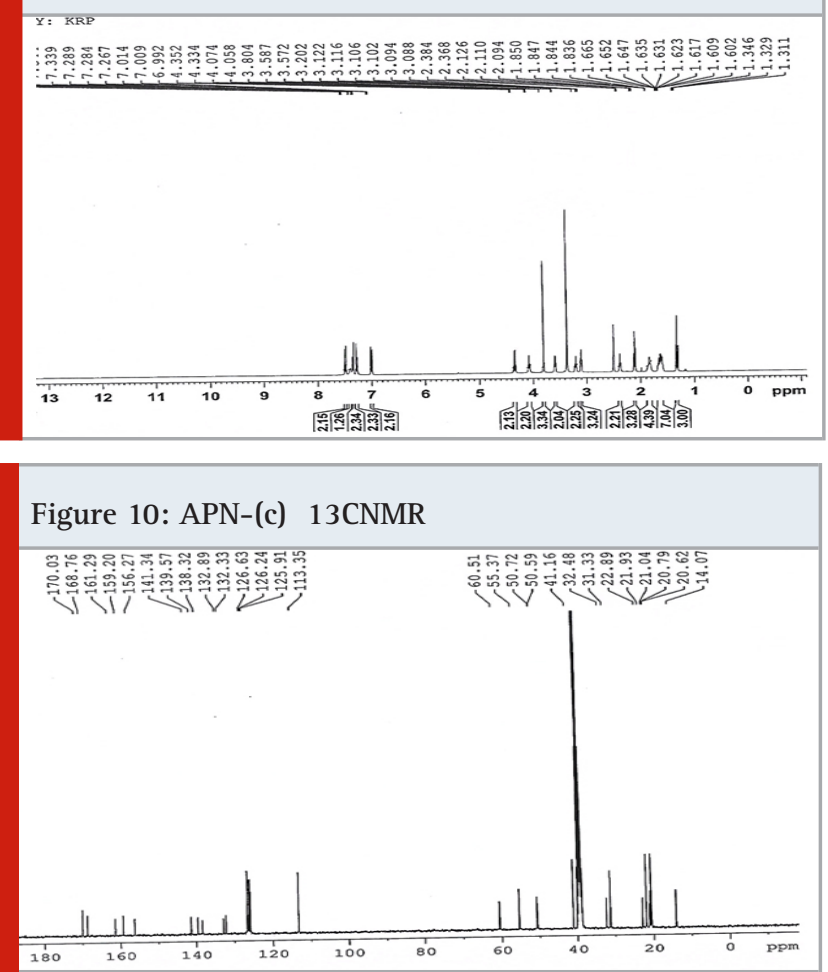

Figure 11: APN-(c) FT-IR

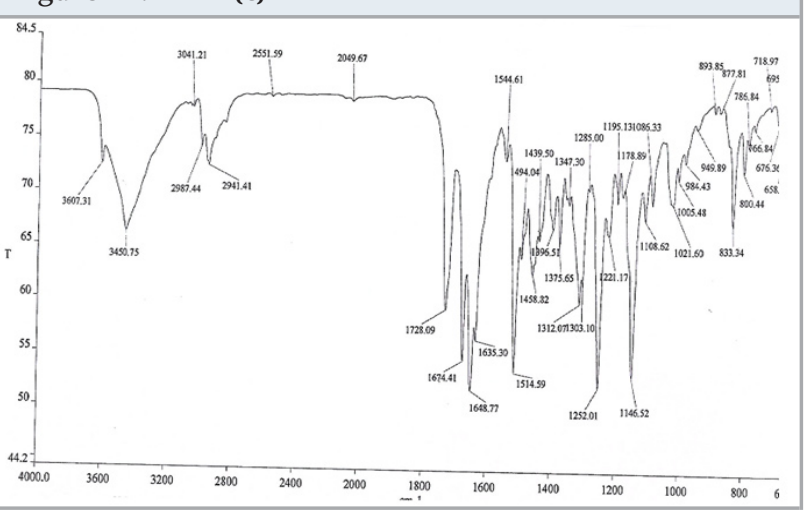


Figure 12: APN-(c) MASS

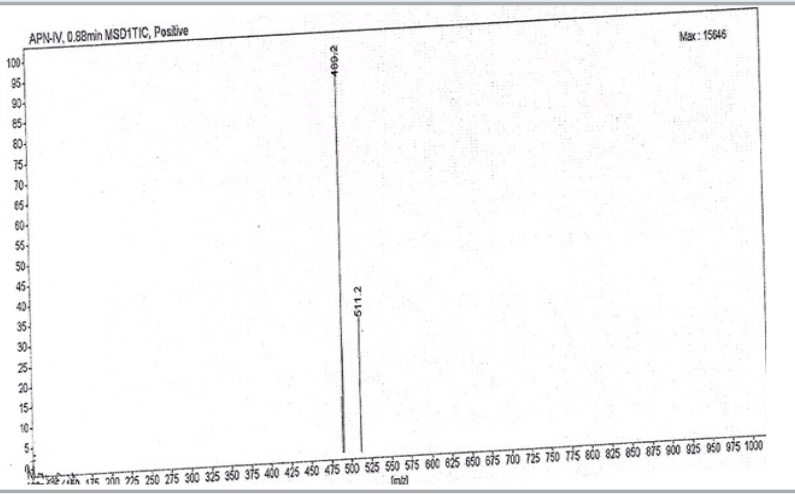

Figure 13: APN-(d) 1HNMR

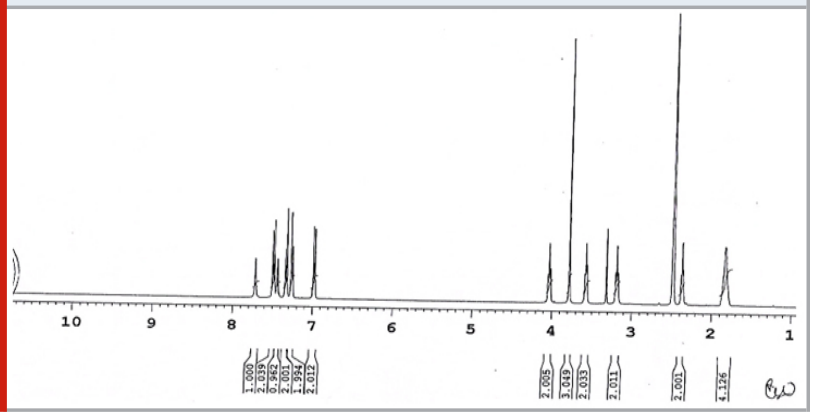

Figure 14: APN-(d) FT-IR

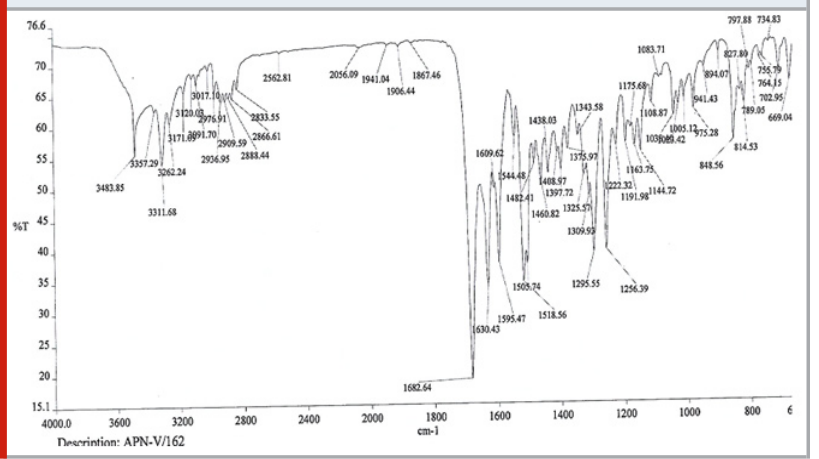

Figure 15: APN-(d) 13CNMR

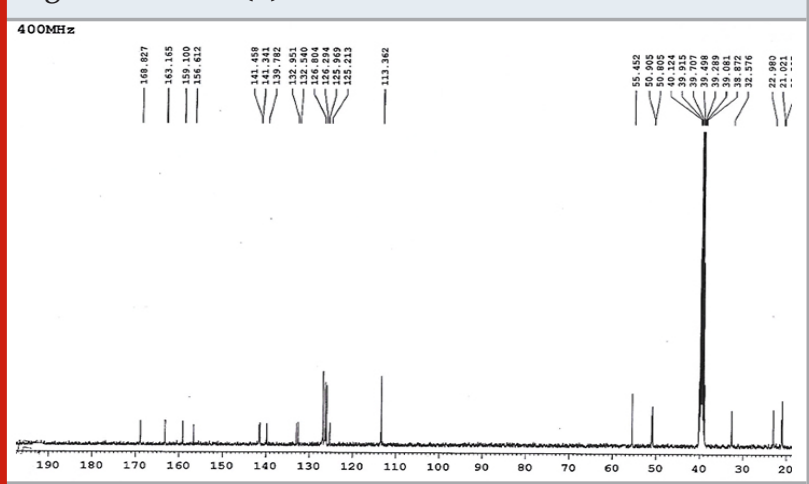

Step-5: Ethyl-1-(4-Methoxyphenyl)-7-0xo-6-(4-(2Oxopiperidin-1yl) Phenyl)-4, 5, 6, 7-Tetrahydro-1hPyrazolo[3,4c]Pyridine-3-Carboxamide(d): In a 250 $\mathrm{mL}$ round bottom flask taken $12 \mathrm{gm}$ of Sodamide and
Methylene dichloride (MDC)(250 mL) and then added 30 gm Ethyl-1-(4-methoxyphenyl)-7-oxo-6-(4-(2oxopiperidin-1yl)phenyl)-4, 5, 6, 7-tetrahydro-1Hpyrazolo[3,4c]pyridine-3-carboxylate. The above reaction mass was cooled to $-50 \mathrm{C}$, added $12.1 \mathrm{gm}$ of sodium methoxide powder lot wise in 45 minutes duration. After addition was completed reaction temperature rose to room temperature and maintains for 1-2 hours. After completion of the reaction, the reaction mass was filtered, the filtered cake was washed with MDC $(125 \mathrm{~mL})$ and the filtrate MLs was washed with Water, the organic layer was concentrated and the resulting solid material was isolated in water by filtration. The crude apixaban (23 gm) was purified in methanol to afford $18 \mathrm{gm}$ of pure apixaban with purity of $\leq 99.5 \%$ with single maximum impurity $\leq 0.1 \%$.

1HNMR (CDCl3, ppm): $\delta 1.83(\mathrm{t}, 4 \mathrm{H}) ; \delta 2.38(\mathrm{t}, 2 \mathrm{H}) ; \delta 3.20$ $(\mathrm{t}, 2 \mathrm{H}) ; \delta 3.58(\mathrm{t}, 2 \mathrm{H}) ; \delta 3.79(\mathrm{~s}, 3 \mathrm{H}) ; \delta 4.04(\mathrm{t}, 2 \mathrm{H}) ; \delta 7.00$ $(\mathrm{d}, 2 \mathrm{H}) ; \delta 7.28(\mathrm{~d}, 2 \mathrm{H}) ; \delta 7.33(\mathrm{~d}, 2 \mathrm{H}) ; \delta 7.44(\mathrm{~s}, 1 \mathrm{H}) ; \delta 7.49$ (d, 2H); $\delta 7.71$ (s, 1H); 13C NMR(ppm): 21.00, 22.97, 32.56, 50.79, 55.44, 113.35, 125.18, 125.96, 126.79, 132.53, 132.94, 139.77,141.44, 156.60, 159.09, 163.14, 168.80; Mass: $460.20(\mathrm{M}+\mathrm{H})$; FT-IR(Cm-1): 3311, 3484, 3017, 3071, 2930, 2977, 1595, 1630, 1683, 1144, 1256, 1296.

\section{CONCLUSION}

In conclusion, the present paper summarizes the improved synthetic approach utilized for the synthesis of the anticoagulant drug apixaban. The synthetic approach of apixaban was made by using commercially available chlorinating agent sulfurylchloride that a new amidation process for ester simply by treating with sodamide and sodium methoxide in methylene dichloride. The developed process could be used for the industrial production of apixaban in high yield and enantio purity.

\section{ACKNOWLEDGEMENTS}

The authors are thankful to Department of Chemistry, RVR \& JC Engineering College, Guntur, A.P, India for providing encouraging environment and facilities for research work.

\section{REFERENCES}

Ji, Y., Liu, Q., Liuai,X., Jiang, J., Wang, Y., Wang C., Yuyan, K.2012. Method for preparing antithrombotic medicament apixaban. CN. Patent 201010277358 .July 04, (2012).

Apixaban. Monograph for Professionals.2019. American Society of Health-System Pharmacists.

Dwivedi SD., Singh KK., Tandon N., Ware D. 2014.An improved process for the preparation of apixaban and intermediates .WO. Patent 2014/203275,

Guo, F., Renli, X., Xu, He L.2014. A method of preparing intermediates of apixaban CN. Patent. 201410113371.

Huo, SW., Guo, KY., Zhong,, J., When, HL.2014. Preparation method of apixaban intermediates. CN. 
Patent 201210305258.

Jianan Jiang., Yafei, Ji..2012. Alternate Synthesis of Apixaban (BMS-562247), an Inhibitor of Blood Coagulation Factor Xa,An International Journal for Rapid Communication of Synthetic Organic Chemistry, 43(1):72-79

Raghavan, N., Frost, CE., Yu, Z., He, K., Zhang, H., Humphreys, WG., Pinto D., Chen S., Bonacorsi ,S., Wong, PC., Zhang. D. 2009. Apixaban metabolism and pharmacokinetics after oral administration to humans. Drug Metabolism and Disposition. 37(1):74-81. ISSN:0090-9556.

Shekar Bhaskar Bhirud., Sushanta Mishra., Suresh Babu Narayanan.2014. Process for the preparation and purification of apixaban, WO 2014111954 A1.

Zhou , JC., Oh, LM., Ma, P., Li , HY. 2003.Synthesis of 4,5-dihydro-pyrazolo[3,4-c]pyrid-2-ones. WO Patent 2003/0 49681. 